\title{
Dermoscopy of squamoid eccrine ductal carcinoma: an aid for early diagnosis*
}

\author{
Márcio Martins Lobo-Jardim, Bruno de Castro e Souza ${ }^{1}$, Priscila Kakizaki, \\ Neusa Yurico Sakai Valente ${ }^{1}$
}

DOI: http://dx.doi.org/10.1590/abd1806-4841.20187307

\begin{abstract}
Squamoid eccrine ductal carcinoma is a cutaneous malignancy that originates from the eccrine sweat gland. A 76-year-old woman presented with an asymptomatic papule on her nose that had been present for one year. Dermoscopy showed pseudocysts, irregular linear crown vessels, and yellowish-brown globules surrounded by white halos. This is the first dermoscopic description of squamoid eccrine ductal carcinoma. Studies with more cases are needed to confirm the dermoscopic characterization of this tumor.
\end{abstract}

Keywords: Clinical diagnosis; Dermoscopy; Skin neoplasms

Squamoid eccrine ductal carcinoma (SEDC) is a rare malignant neoplasm of the skin that arises from the eccrine gland and presents on histology with a squamoid appearance, potentially mistaken for squamous cell carcinoma. ${ }^{1}$

The clinical importance of SEDC lies in its locoregional aggressiveness and metastatic potential. However, the clinical presentation is sometimes nonspecific or displays a benign appearance, characteristics that can delay diagnosis. ${ }^{2}$ We thus report a case of SEDC, the main focus of which is to describe the dermoscopic characteristics with a view towards earlier clinical diagnosis of these lesions.

A 76-year-old female patient presented with an asymptomatic lesion on the nose that had appeared a year before. Her medical history included hypertension, osteoporosis, and Bowen's disease, excised in 2014. Clinical examination showed a well-demarcated normochromic lesion on the nasal dorsum (Figure 1). Dermoscopy revealed a circumscribed lesion with pseudocysts (or white lumps), irregular linear vessels not extending beyond the center of the lesion, and yellowish-brown globules (or lumps) surrounded by white halos located close to the linear vessels (Figure 2). An excisional biopsy was performed, submitted to hematoxylin \& eosin staining, showing an infiltrative malignant epithelial neoplasm, connected to the epidermis and to the hair follicles, with blocks of squamoid cells above and small blocks of smaller cells below (Figures 3 and 4 ), with ducts in both components. The deep margin represented by the reticular dermis was involved, and the lateral margins were free. Immunohistochemistry with antibodies to the carcinoembryonic antigen and epithelial membrane antigen was positive in the areas of ductal differentiation, corroborating the diagnosis of SEDC. Following diagnosis, extension of the surgical resection was performed.

SEDC is a rare neoplasm of the skin, described for the first time in 1997 by Wong et al. It represents $0.01 \%$ of all malignant cutaneous neoplasms. Histologically, SEDC presents a pattern of infil-

\footnotetext{
Received 22 May 2017.

Accepted 01 January 2018.

* Work conducted at Hospital do Servidor Público Estadual de São Paulo, São Paulo (SP), Brazil.

Financial support: None.

Conflict of interest: None.

1 Dermatology Service, Hospital do Servidor Público Estadual de São Paulo, São Paulo (SP), Brazil.
}

MaILING AdDRESS:

Márcio Martins Lobo Jardim

E-mail: martinslobojardim@gmail.com

(C2018 by Anais Brasileiros de Dermatologia 


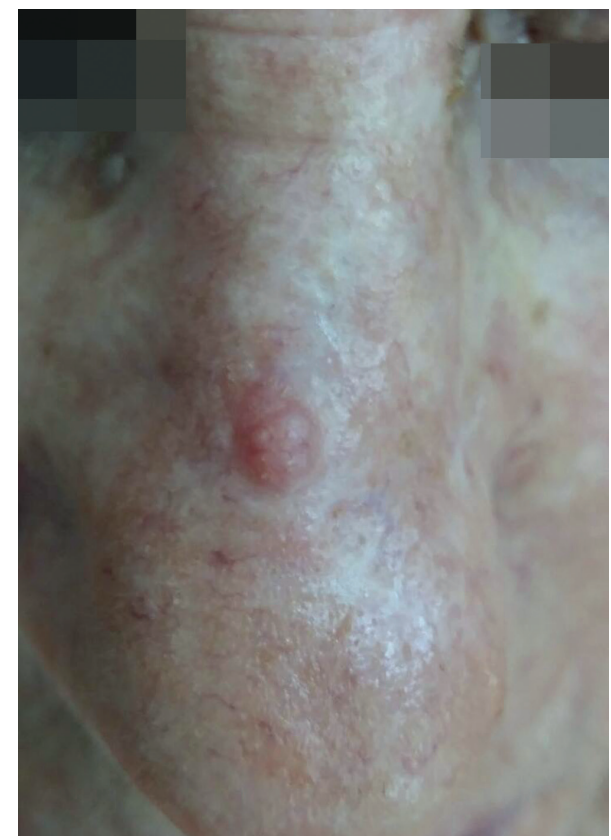

Figure 1:

Normochromic papule located on the nasal dorsum

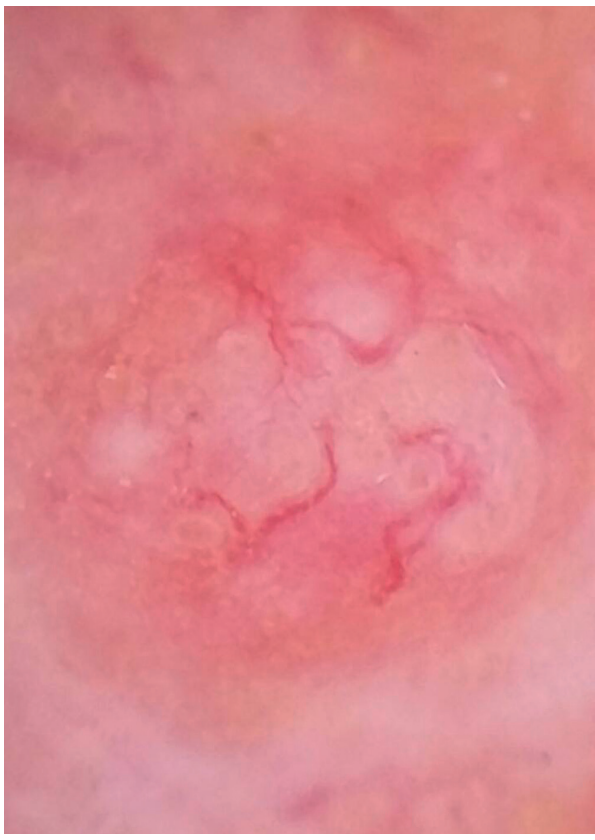

FigURE 2:

Dermoscopy of the lesion shows irregular linear vessels, pseudocysts, and yellowishbrown lumps (globules) surrounded by white halos

trative growth, poorly demarcated, extending deeply to the dermis and subcutaneous tissue. The lesion's surface typically presents a squamoid appearance and ductal differentiation in the deep portion. In $86 \%$ of cases there are associated actinic keratoses, similar to the case described here. ${ }^{1,2}$

The clinical relevance of SEDC lies in its metastatic potential and locoregional aggressiveness, despite traditionally being considered a low-grade neoplasm. In 2016, Van der Horst et al. analyzed a series of 30 cases and found a $25 \%$ local recurrence rate and $13 \%$

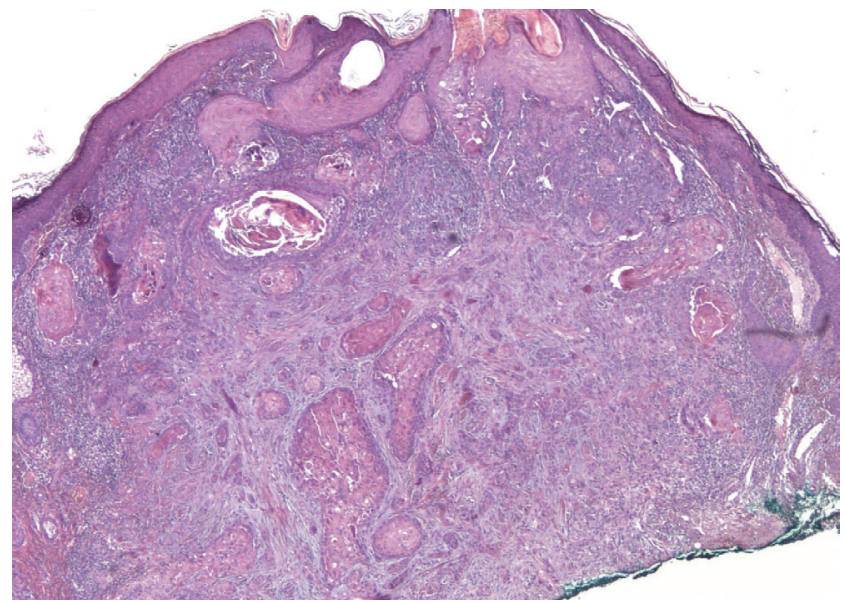

FIGURE 3: Malignant epithelial neoplasm with squamoid areas, ductal differentiation, connected to the epidermis and hair follicles, infiltrative and desmoplastic (Hematoxylin \& eosin, $x 40$ )

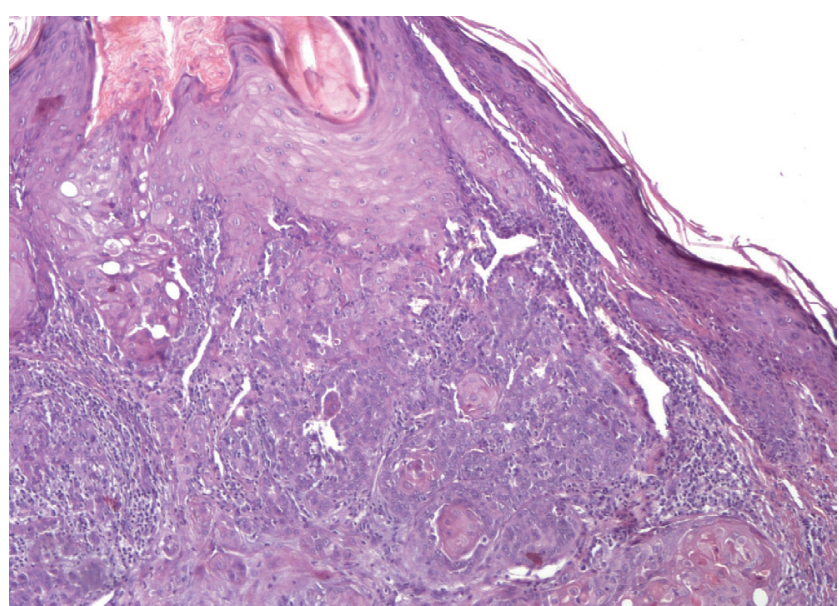

FIGURE 4: Central area with neoplastic proliferation associated with hair follicles. Periphery with solar elastosis and vascular ectasias (Hematoxylin \& eosin, x100)

metastasis rate, with three cases of lymph node metastasis and one distant metastasis. ${ }^{2}$

The lesions are located on the face or neck in the majority of cases, although the trunk and extremities may be involved. As for clinical characteristics, the lesions present as papules, nodes, or plaques, which can be ulcerated or even simulate benign lesions. This clinical non-specificity often leads to incomplete biopsies (shavings), diagnostic delay, and negative impact on prognosis. ${ }^{2,3}$

In this context, a dermoscopic analysis of SEDC can be useful for early diagnosis, although it has not been reported in the literature. The case described here features the presence of yellowish-brown globules (or lumps) surrounded by white halos which on histology were associated with follicular ostia surrounded by tumor proliferation in the epidermis and dermis. Analogously, the presence of white halos has been described both in porocarcinoma and in well-differentiated squamous cell carcinoma, with similar 
histological correlates. However, unlike SEDC, in these neoplasms, the white halos include red globules or dots due to the presence of a richly vascularized dermal stroma. ${ }^{4,5}$

On histology, the irregular linear vessels correspond to dilated superficial vessels limited to the lesion's periphery without crossing the tumor mass. This vascular pattern is also found in sebaceous hyperplasia and is known in the literature as "crown vessels". However, besides this vascular pattern, sebaceous hyperplasia also displays yellow globules in the lesion's center, but without the white halo, an important characteristic for performing differential diagnosis with SEDC, in which the presence of white halos surrounding yellowish-brown globules results from the tumor proliferation in the dermis and epidermis.
The pseudocysts (or white lumps) found in the case reported here were associated with the presence of corneal cysts in the dermis.

Interpreting these findings, we suggest the possibility that they were influenced by the fact that the lesion was located on the face, where there are abundant hair follicles, possibly explaining the presence of pseudocysts and yellowish-brown globules (or lumps).

Finally, this is the first dermoscopic description of squamoid eccrine ductal carcinoma in the literature. Further publications with dermoscopic descriptions of SEDC are necessary, especially when the carcinoma is located on the trunk and extremities. Such publications could confirm and complement the dermoscopic findings reported in the current case. $\square$

\section{REFERENCES}

1. Wong TY, Suster S, Mihm MC.. Squamoid eccrine ductal carcinoma. Histopathology. 1997;30:288-93.

2. van der Horst MP, Garcia-Herrera A, Markiewicz D, Martin B, Calonje E, Brenn T. Squamoid Eccrine Ductal Carcinoma: A Clinicopathologic Study of 30 Cases. Am J Surg Pathol. 2016;40:755-60.

3. Saraiva MI, Vieira MA, Portocarrero LK, Fraga RC, Kakizaki P, Valente NY. Squamoid eccrine ductal carcinoma. An Bras Dermatol. 2016;91:799-802.
4. Zalaudek I, Argenziano G. Dermoscopy of actinic keratosis, intraepiderma carcinoma and squamous cell carcinoma. Curr Probl Dermatol. 2015;46:70-6.

5. Edamitsu T, Minagawa A, Koga H, Uhara H, Okuyama R. Eccrine porocarcinoma shares dermoscopic characteristics with eccrine poroma: A report of three cases and review of the published work. J Dermatol. 2016;43:332-5.

AUTHORS'CONTRIBUTIONS
Márcio Martins Lobo Jardim
Conception and planning of the study, Elaboration and writing of the manuscript, Obtaining, analyzing and interpreting the data, Intellectual participation in propaedeutic and/or thera-
peutic conduct of the cases studied, Critical review of the literature, Critical review of the manuscript
Bruno de Castro e Souza
Conception and planning of the study, Obtaining, analyzing and interpreting the data, Critical review of the literature, Critical review of the manuscript
Priscila Kakizaki
Obtaining, analyzing and interpreting the data, Intellectual participation in propaedeutic and/or therapeutic conduct of the cases studied, Critical review of the literature, Critical review
of the manuscript
$\begin{aligned} & \text { Neusa Yurico Sakai Valente } \\ & \text { Intellectual participation in propaedeutic and/or therapeutic conduct of the cases studied, Critical review of the literature, Critical review of the manuscript }\end{aligned}$

How to cite this article: Lobo-Jardim MM, Souza BC, Kakizaki P, Valente NYS. Dermoscopy of squamoid eccrine ductal carcinoma: an aid for early diagnosis. An Bras Dermatol. 2018;93(6):893-5. 